\title{
Article \\ Periodic Points of Modular Firmly Mappings in the Variable Exponent Sequence Spaces $\ell_{p(\cdot)}$
}

\author{
Afrah A. N. Abdou ${ }^{1}$ and Mohamed A. Khamsi ${ }^{2, *(1)}$ \\ 1 Department of Mathematics, Faculty of Sciences, University of Jeddah, Jeddah 21589, Saudi Arabia; \\ aabdou@uj.edu.sa \\ 2 Department of Applied Mathematics and Sciences, Khalifa University, \\ Abu Dhabi 127788, United Arab Emirates \\ * Correspondence: mohamed.khamsi@ku.ac.ae
}

check for updates

Citation: Abdou, A.A.N.; Khamsi, M.A. Periodic Points of Modular Firmly Mappings in the Variable Exponent Sequence Spaces $\ell_{p(\cdot)}$. Mathematics 2021, 9, 2418. https:// doi.org/10.3390/math9192418

Academic Editor: Christopher Goodrich

Received: 26 August 2021

Accepted: 25 September 2021

Published: 28 September 2021

Publisher's Note: MDPI stays neutral with regard to jurisdictional claims in published maps and institutional affiliations.

Copyright: (C) 2021 by the authors. Licensee MDPI, Basel, Switzerland. This article is an open access article distributed under the terms and conditions of the Creative Commons Attribution (CC BY) license (https:/ / creativecommons.org/licenses/by/ $4.0 /)$.

\begin{abstract}
In this work, we investigate the existence of periodic points of mappings defined on nonconvex domains within the variable exponent sequence spaces $\ell_{p(\cdot)}$. In particular, we consider the case of modular firmly nonexpansive and modular firmly asymptotically nonexpansive mappings. These kinds of results have never been obtained before.
\end{abstract}

Keywords: electrorheological fluids; fixed point; modular firmly nonexpansive mapping; modular vector spaces; periodic point; strictly convex; uniformly convex

MSC: primary 47H09; 47H10

\section{Introduction}

A central concept in nonlinear analysis and optimization is that of a firmly nonexpansive mapping, mainly due to its relation with maximally monotone operators, as evidenced in [1-5] and the references therein. In 1962, Minty [6,7] implicitly employed the idea of firmly nonexpansive mappings to study monotone mappings. The definition of firmly nonexpansive mappings on Banach spaces was introduced by Bruck [8] in 1973. The fixed point results for such mappings obtained by Kaczor [9] stand out among others of their kind. In fact, Kaczor's results may be seen as a wonderful extension to Smarzewski's work [10].

In principle, the concept of firm nonexpansiveness can be carried over to the case of modular spaces. The first attempt in this direction is due to Khan [11].

This work delves deeper into firm nonexpansiveness in the modular setting; specifically, we recover most of the results by [9] for mappings defined on the variable exponent sequence spaces $\ell_{p(\cdot)}$.

These spaces originated from the consideration of certain problems related to Fourier series in the work by Orlicz [12]. The study of these spaces was furthered in [13-16]; they played a motivational role in the introduction of the concept of modular [17]. Moreover, the above mentioned sequence spaces are the discrete version of the Lebesgue variable exponent spaces $L^{p(.)}$. In recent years, these spaces took an important role in the study of many problems for which the classical $L^{p}$ spaces are not appropriate to be used. Due to their importance in applications, the variable exponent Lebesgue spaces became a major area of mathematical work [18]. In particular, it is worth noticing the importance of these spaces in the treatment of the hydrodynamical behavior of non-Newtonian fluids $[19,20]$ and its applications to several areas of Engineering and Medicine [21-24].

In this work, we investigate the existence of periodic points for firmly asymptotically nonexpansive mappings defined within the spaces $\ell_{p(\cdot)}$. In particular, we extend Kaczor's results [9] in the modular sense. As was noted in [25], the main major issue with the results of [11] is the assumption made by the author about the domain being convex. 
Our work requires the tools from the field of fixed point theory, for which the reader is referred to the books [26-28].

\section{Basic Definitions \& Results}

We start this section by summarizing some simple facts about the linear spaces $\ell_{p(\cdot)}$.

Definition 1 ([12]). the linear spaces $\ell_{p(\cdot)}$ are defined as

$$
\ell_{p(\cdot)}=\left\{\left(x_{n}\right) \subset \mathbb{R}^{\mathbb{N}} ; \sum_{n=0}^{\infty} \frac{1}{p(n)}\left|\frac{x_{n}}{\beta}\right|^{p(n)}<+\infty, \text { for some } \beta>0\right\},
$$

where $p: \mathbb{N} \rightarrow[1, \infty)$.

In [12], Orlicz introduced these spaces using a slightly different terminology and notation. These spaces inspired Nakano who developed a more general theory known as modular vector spaces $[14,17,29]$.

Proposition $1([13,15,17])$. On $\ell_{p(\cdot)}$, we introduce the functional $\varrho: \ell_{p(\cdot)} \rightarrow[0, \infty]$ by

$$
\varrho(u)=\varrho\left(\left(u_{n}\right)\right)=\sum_{n=0}^{\infty} \frac{1}{p(n)}\left|u_{n}\right|^{p(n)} .
$$

The function $\rho$ has the following properties:

(i) $\varrho(u)=0$ if and only if $u=0$;

(ii) $\varrho( \pm u)=\varrho(u)$;

(iii) $\varrho(\alpha u+(1-\alpha) v) \leq \alpha \varrho(u)+(1-\alpha) \varrho(v)$, for all $\alpha \in[0,1]$,

for any $u, v \in \ell_{p(\cdot)}$.

In addition, $\varrho$ is left-continuous, that is, one has $\lim _{r \rightarrow 1-} \varrho(r w)=\varrho(w)$, for any $w \in \ell_{p(\cdot)}$. Next we adapt to the modular case a few concepts that are commonplace in the metric setting.

Definition $2([25,28])$.

1. A sequence $\left\{u_{n}\right\} \subset \ell_{p(\cdot)}$ is $\varrho$-convergent to $u \in \ell_{p(\cdot)}$ if and only if $\varrho\left(u_{n}-u\right) \rightarrow 0$. It is obvious that, if $Q$-limit exists, then it must be unique;

2. A sequence $\left\{u_{n}\right\} \subset \ell_{p(\cdot)}$ is $\varrho$-Cauchy if $\varrho\left(u_{n}-u_{m}\right) \rightarrow 0$ as $n, m \rightarrow \infty$;

3. A set $C \subset \ell_{p(\cdot)}$ is said to be $\varrho$-closed if for any sequence $\left\{u_{n}\right\} \subset C$ that $\varrho$-converges to $u$, it follows that $u \in C$;

4. $\quad A$ set $C \subset \ell_{p(\cdot)}$ is $\varrho$-bounded if $\delta_{\varrho}(C)=\sup \{\varrho(u-v) ; u, v \in C\}<\infty$;

5. Let $u \in \ell_{p(\cdot)}$ and $R \geq 0$. We define the $Q$-ball centered at $u$ with radius $R$ as

$$
B_{\varrho}(u, R)=\left\{v \in \ell_{p(\cdot)} ; \varrho(u-v) \leq R\right\} .
$$

Moreover, $\varrho$ satisfies the Fatou property, that is to say, if a sequence $\left\{v_{n}\right\} \subseteq \ell_{p(\cdot)}$ $\varrho$-converges to $v$, then, for any $u \in \ell_{p(\cdot)}$, it holds

$$
\varrho(u-v) \leq \liminf _{n \rightarrow \infty} \varrho\left(u-v_{n}\right) .
$$

It is easy to show that the Fatou's property is equivalent to the $\varrho$-closedness of the $\varrho$-balls. We pass to a fundamental property when it comes to modular vector spaces, namely, the $\Delta_{2}$-condition. 
Definition 3. The functional $\varrho$ is said to satisfy the $\Delta_{2}$-condition if there exists $C \geq 0$ such that

$$
\varrho(2 w) \leq C \varrho(w)
$$

for all $w \in \ell_{p(\cdot)}$.

We leave up to the reader the verification of the fact that the modular $\varrho$ satisfies the $\Delta_{2}$-condition if and only if $p^{+}=\sup _{n \in \mathbb{N}} p(n)<\infty[13,15,17]$.

The Luxemburg norm associated to the modular $\varrho$ on $\ell_{p(\cdot)}$ is given by:

$$
\|x\|_{\varrho}=\inf \left\{\lambda>0 ; \varrho\left(\frac{1}{\lambda} x\right) \leq 1\right\} \text {. }
$$

Endowed with the Luxemburg norm, $\left(\ell_{p(\cdot)},\|\cdot\|_{\varrho}\right)$ becomes a Banach space. Most of the geometric properties enjoyed by Banach spaces will hold when $p^{-}=\inf _{n \in \mathbb{N}} p(n)$ and $p^{+}=\sup _{n \in \mathbb{N}} p(n)$ are away from 1 and $+\infty$. In particular, the uniform convexity holds in $\left(\ell_{p(\cdot)},\|\cdot\|_{\varrho}\right)$ if and only if $1<p^{-} \leq p^{+}<+\infty[15]$.

In the next definition, we introduce the class of mappings for which we will investigate the existence of periodic points.

Definition 4. Let $D$ be a nonempty subset of $\ell_{p(\cdot)}$. Fix $\lambda \in(0,1)$. A map $T: D \rightarrow \ell_{p(\cdot)}$ is said to be

1. Ref. [30] Q-nonexpansive, if

$$
\varrho(T(x)-T(x)) \leq \varrho(x-y)
$$

for all $x, y \in D$.

2. Ref. [28] Q-asymptotically nonexpansive, if there exists a sequence of positive numbers $\left\{k_{p}\right\}$ such that $\lim _{p \rightarrow \infty} k_{p}=1$ and

$$
\varrho\left(T^{p}(x)-T^{p}(y)\right) \leq k_{p} \varrho(x-y)
$$

for all $p \geq 1$ and all $x, y \in D$.

3. Ref. $[11,25] \lambda$ - $\varrho$-firmly nonexpansive, if

$$
\varrho(T(x)-T(y)) \leq \varrho((1-\lambda)(x-y)+\lambda(T(x)-T(y)))
$$

for all $x, y \in D$.

4. $\lambda$ - $\varrho$-firmly asymptotically nonexpansive if there exists a sequence of positive numbers $\left\{k_{p}\right\}$ such that $\lim _{p \rightarrow \infty} k_{p}=1$ and

$$
\varrho\left(T^{p}(x)-T^{p}(y)\right) \leq k_{p} \varrho\left((1-\lambda)(x-y)+\lambda\left(T^{p}(x)-T^{p}(y)\right)\right),
$$

for all $p \geq 1$ and all $x, y \in D$.

A fixed point of $T$ is any $x \in D$ such that $T(x)=x$. The set of fixed points of $T$ will be denoted by Fix $(T)$. The fixed points of $T^{n}$ are known as periodic points of $T$, for $n \geq 1$.

The concept of $\lambda$ - $\varrho$-firmly asymptotically nonexpansive mappings is new and was not studied before. The authors of [25] looked at the case of a non convex domain and obtained some interesting results about firmly nonexpansive mappings in the modular sense in $\ell_{p(\cdot)}$. In the next section, we extend their main results to this new class of mappings. 


\section{Main Results}

Periodic points are a special case of the fixed point property. Most of the interesting results in this area use some kind of geometric properties. This is the reason many authors worked on establishing the modular version of these geometric properties, such as strict convexity, uniform convexity and so forth (see, for example, refs. [28,31,32]).

The following definition was introduced in [25] and proved to be useful.

Definition 5. Let $D$ be a nonempty subset of $\ell_{p(\cdot)}$. We will say that $D$ is $\varrho$-connected if and only if $D$ is not a finite union of disjoint $Q$-closed nonempty subsets.

Note that $\varrho$-closed convex subsets of $\ell_{p(\cdot)}$ are $\varrho$-connected. Next, recall that a subset $K$ of $\ell_{p(\cdot)}$ is said to have the fixed point property for $\varrho$-nonexpansive self-mappings if any $\varrho$-nonexpansive mapping $S: K \rightarrow K$ has a fixed point. Convex subsets of $\ell_{p(\cdot)}$ having the fixed point property for $\varrho$-nonexpansive self-mappings were extensively studied [28]. Note that in [32], it is proved that if $\{n \in \mathbb{N} ; p(n)=1\}$ has at most one element, then any nonempty $\varrho$-bounded convex $\varrho$-closed subset $K$ of $\ell_{p(\cdot)}$ has the fixed point property for $Q$-nonexpansive mappings provided $K$ enjoys the property $(R)$. This property is very similar to the reflexivity or weak-compactness in Banach spaces. Recall that $K$ enjoys the property (R) if and only if for any decreasing sequence $\left\{K_{n}\right\}_{n \geq 1}$ of $\varrho$-closed, convex nonempty subsets of $K$, has a nonempty intersection.

Theorem 1. Let $K$ be a union of nonempty, pairwise disjoint, $\varrho$-closed and $\varrho$-connected subsets $K_{1}, K_{2}, \cdots, K_{n}$ of $\ell_{p(\cdot),}$ with $n \geq 2$. Assume that one of the subsets $K_{1}, K_{2}, \cdots, K_{n}$ has the fixed point property for $Q$-nonexpansive self-mappings. Then any $Q$-nonexpansive mapping $S: K \rightarrow K$ has a periodic point.

Proof. Since $S$ is $\varrho$-nonexpansive, then $S^{-1}\left(K_{i}\right)$ is $\varrho$-closed, for any $i=1, \cdots, n$. Clearly, we have $K_{j}=\bigcup_{i=1}^{n} S^{-1}\left(K_{i}\right) \cap K_{j}$. Since $\left\{S^{-1}\left(K_{i}\right)\right\}$ are $\varrho$-closed disjoint subsets and each $K_{j}$ is $\varrho$-connected, we deduce that, for any $i \in\{1, \cdots, n\}$, there exists $k \in\{1, \cdots, n\}$ such that $S\left(K_{i}\right) \subset K_{k}$. Without loss of any generality, we assume that $K_{1}$ has the fixed point property for $\varrho$-nonexpansive self-mappings. Therefore, there exists $p \in\{1, \cdots, n\}$ such that $S^{p}\left(K_{1}\right) \subset K_{1}$. Since $S^{p}$ is $\varrho$-nonexpansive, we conclude that $S^{p}$ has a fixed point in $K_{1}$, that is, $T$ has a periodic point in $K_{1}$.

In general, there is no reason for the periodic point to be a fixed point of the mapping. If we assume that the modular functional $\varrho$ satisfies some geometric properties, then this will hold. This is the main driving argument behind the main results of [25].

Theorem 2 ([25]). Assume $\{n \in \mathbb{N} ; p(n)=1\}$ has at most one element. Let $C=\bigcup_{i=1}^{n} C_{i}$, with $n \geq 2$, be a union of nonempty, pairwise disjoint, $\varrho$-closed and $\varrho$-connected subsets $C_{i}$ of $\ell_{p(\cdot)}$.

(1) Assume that $C_{1}$ has the fixed point property for $\varrho$-nonexpansive self-mappings. If for some $\lambda \in(0,1), T: C \rightarrow C$ is $\lambda$ - $\varrho$-firmly nonexpansive, then $T$ has a fixed point in $C$.

(2) Assume that $C_{1}$ is $\varrho$-bounded, convex and has the property (R). If for some $\lambda \in(0,1)$, $T: C \rightarrow C$ is $\lambda$ - $Q$-firmly nonexpansive, then $T$ has a fixed point in $C$.

As we said earlier, the main results of our work are the extension of the above conclusions to $\lambda$ - $\varrho$-firmly asymptotically nonexpansive mappings. The following technical simple fact will be useful.

Lemma 1. Let $C$ be a nonempty subset of the vector space $\ell_{p(\cdot)}$. If for some $\lambda \in(0,1), T$ : $C \rightarrow C$ is $\lambda$-Q-firmly asymptotically nonexpansive, then there exists $p_{0} \geq 1$, such that $T^{p}$ is $\varrho$-asymptotically nonexpansive, for any $p \geq p_{0}$. 
Proof. Since $T$ is $\lambda$-firmly asymptotically nonexpansive, there exists a sequence of positive numbers $\left\{k_{p}\right\}$ such that $\lim _{p \rightarrow \infty} k_{p}=1$ and

$$
\varrho\left(T^{p}(x)-T^{p}(y)\right) \leq k_{p} \varrho\left((1-\lambda)(x-y)+\lambda\left(T^{p}(x)-T^{p}(y)\right)\right),
$$

for any $x, y \in C$ and $p \in \mathbb{N}$. Since $\lim _{p \rightarrow \infty} \lambda k_{p}=\lambda<1$, there exists $p_{0} \geq 1$ such that $\lambda k_{p}<1$, for any $p \geq p_{0}$. Using the fact that $\varrho$ is convex, we get

$$
\varrho\left(T^{p}(x)-T^{p}(y)\right) \leq k_{p}(1-\lambda) \varrho(x-y)+k_{p} \lambda \varrho\left(T^{p}(x)-T^{p}(y)\right),
$$

which implies

$$
\varrho\left(T^{p}(x)-T^{p}(y)\right) \leq \frac{k_{p}(1-\lambda)}{1-\lambda k_{p}} \varrho(x-y),
$$

for $p \geq p_{0}$ and $x, y \in C$. Since

$$
\lim _{p \rightarrow \infty} \frac{k_{p}(1-\lambda)}{1-\lambda k_{p}}=1
$$

we conclude that $T^{p}$ is $\varrho$-asymptotically nonexpansive, for any $p \geq p_{0}$.

Before we state our next result on $\varrho$-asymptotically nonexpansive mappings, we recall that if $p^{-}=\inf _{n \in \mathbb{N}} p(n)>1$, then $\varrho$ enjoys a modular uniform convexity [31], which will secure that any nonempty $\varrho$-bounded convex $\varrho$-closed subset $C$ of $\ell_{p(\cdot)}$ has the fixed point property for $\varrho$-asymptotically nonexpansive self-mappings [28].

Theorem 3. Assume $p^{-}>1$. Let $K=\bigcup_{i=1}^{n} K_{i}$, with $n \geq 2$, are a union of nonempty, pairwise disjoint, $\varrho$-closed and $\varrho$-connected subsets $K_{i}$ of $\ell_{p(\cdot)}$. Assume that $K_{1}$ is a nonempty $\varrho$-bounded convex $\varrho$-closed subset $C$ of $\ell_{p(\cdot)}$. If, for some $\lambda \in(0,1), T: K \rightarrow K$ is $\lambda$ - $\varrho$-firmly asymptotically nonexpansive, then $T$ has a periodic point in $K$.

Proof. Lemma 1 implies the existence of $p_{0} \geq 1$ such that $T^{p}$ is $\varrho$-asymptotically nonexpansive, for any $p \geq p_{0}$. Fix $p>p_{0}$. Set $S=T^{p}$. Using the same argument in the proof of Theorem 1, there exists $n \geq 1$ such that $S^{n}\left(K_{1}\right) \subset K_{1}$, i.e., $T^{n p}\left(K_{1}\right) \subset K_{1}$. Since $K_{1}$ has the fixed point property for $\varrho$-asymptotically nonexpansive self-mappings, we conclude that $T^{n p}$ has a fixed point in $K_{1}$, which is a periodic point of $T$.

A similar result to Theorem 2 for $\lambda$ - $\varrho$-firmly asymptotically nonexpansive mappings is the following:

Theorem 4. Assume $p^{-}>1$. Let $K=\bigcup_{i=1}^{n} K_{i}$, with $n \geq 2$, be a union of nonempty, pairwise disjoint, $\varrho$-closed and $\varrho$-connected subsets $K_{i}$ of $\ell_{p(\cdot)}$. Assume that $K_{1}$ is a nonempty $\varrho$-bounded convex $\varrho$-closed subset $K$ of $\ell_{p(\cdot)}$. If for some $\lambda \in(0,1), T: K \rightarrow K$ is $\lambda$ - $Q$-firmly asymptotically nonexpansive, then $\mathrm{T}$ has a fixed point in $\mathrm{K}$.

Proof. Using Theorem 3 , there exists a periodic point $a_{0} \in K$ of $T$, that is, $T^{q}\left(a_{0}\right)=a_{0}$, for some $q \geq 1$. Without loss of generality, we may assume $q \geq 2$. Since $T$ is $\lambda$ - - -firmly asymptotically nonexpansive, there exists a sequence of positive numbers $\left\{k_{p}\right\}$ such that $\lim _{p \rightarrow \infty} k_{p}=1$ and

$$
\varrho\left(T^{p}(a)-T^{p}(b)\right) \leq k_{p} \varrho\left((1-\lambda)(a-b)+\lambda\left(T^{p}(a)-T^{p}(b)\right)\right),
$$


for any $a, \in K$ and $p \in \mathbb{N}$. Set $a_{i}=T^{i}\left(a_{0}\right)$, for $i \geq 1$. We have $a_{i+q}=a_{i}$, for $i \in \mathbb{N}$, which implies $T^{p q}\left(a_{i}\right)=a_{i+p q}=a_{i}$, for any $i, p \in \mathbb{N}$. Hence for any $i, j \geq 1$ and $p \in \mathbb{N}$, we have

$$
\begin{aligned}
\varrho\left(T\left(a_{i}\right)-T\left(a_{j}\right)\right) & =\varrho\left(T^{p q+1}\left(a_{i}\right)-T^{p q+1}\left(a_{j}\right)\right) \\
& \leq k_{p q+1} \varrho\left((1-\lambda)\left(a_{i}-a_{j}\right)+\lambda\left(T^{p q+1}\left(a_{i}\right)-T^{p q+1}\left(a_{j}\right)\right)\right) \\
& \leq k_{p q+1} \varrho\left((1-\lambda)\left(a_{i}-a_{j}\right)+\lambda\left(T\left(a_{i}\right)-T\left(a_{j}\right)\right)\right) .
\end{aligned}
$$

If we let $p \rightarrow \infty$ and using $\lim _{n \rightarrow \infty} k_{n}=1$, we get

$$
\varrho\left(T\left(a_{i}\right)-T\left(a_{j}\right)\right) \leq \varrho\left((1-\lambda)\left(a_{i}-a_{j}\right)+\lambda\left(T\left(a_{i}\right)-T\left(a_{j}\right)\right)\right),
$$

which implies $\varrho\left(T\left(a_{i}\right)-T\left(a_{j}\right)\right) \leq \varrho\left(a_{i}-a_{j}\right)$, for any $i, j \geq 1$. Moreover, we have

$$
\varrho\left(a_{i}-a_{j}\right)=\varrho\left(a_{i+q}-a_{j+q}\right) \leq \varrho\left(a_{i+1}-a_{j+1}\right)=\varrho\left(T\left(a_{i}\right)-T\left(a_{j}\right)\right),
$$

since $q \geq 2$, which implies

$$
\varrho\left(a_{i+1}-a_{j+1}\right)=\varrho\left(T\left(a_{i}\right)-T\left(a_{j}\right)\right)=\varrho\left(a_{i}-a_{j}\right),
$$

for any $i, j \geq 1$. In other words, the restriction of $T$ to the set $\left\{a_{0}, a_{1}, \cdots, a_{q-1}\right\}$ is a $\varrho$ isometry. Define $T_{\lambda}=(1-\lambda) I+\lambda T$, where $I$ is the identity map. Using the inequality $(F N)$, we get

$$
\varrho\left(a_{i}-a_{j}\right)=\varrho\left(T\left(a_{i}\right)-T\left(a_{j}\right)\right) \leq \varrho\left(T_{\lambda}\left(a_{i}\right)-T_{\lambda}\left(a_{j}\right)\right) \leq \varrho\left(a_{i}-a_{j}\right),
$$

for any $i, j \geq 1$. Hence, the restriction of $T_{\lambda}$ to the set $\left\{a_{0}, a_{1}, \cdots, a_{q-1}\right\}$ is also a $\varrho$-isometry. Fix $i \in[1, q-1]$ and set $u=a_{i}-a_{i-1}$ and $v=T\left(a_{i}\right)-T\left(a_{i-1}\right)=a_{i+1}-a_{i}$. Note that $\varrho(u)=\varrho(v)$. Moreover, we have:

$$
\begin{aligned}
\varrho((1-\lambda) u+\lambda v) & =\varrho\left(T_{\lambda}\left(a_{i}\right)-T_{\lambda}\left(a_{i-1}\right)\right) \\
& =\varrho\left(a_{i}-a_{i-1}\right) \\
& =(1-\lambda) \varrho(u)+\lambda \varrho(v) .
\end{aligned}
$$

Our assumption on $p(\cdot)$ implies $\varrho$ is (SC) [32], which implies $u=v$, that is, $a_{i}-a_{i-1}=$ $a_{i+1}-a_{i}$. Since $i$ was taken arbitrarily, we get

$$
a_{1}-a_{0}=a_{2}-a_{1}=\cdots=a_{p-1}-a_{p-2}=a_{q}-a_{q-1},
$$

which implies

$$
q\left(a_{1}-a_{0}\right)=\sum_{i=0}^{q-1} a_{i+1}-a_{i}=a_{q}-a_{0}=a_{0}-a_{0}=0 .
$$

In other words, we have $T\left(a_{0}\right)=a_{1}=a_{0}$, that is, $a_{0}$ is a fixed point of $T$.

It is not known under what general conditions the periodic point discovered in Theorem 3 is a fixed point. However, if $\varrho$ satisfies the triangle inequality, that is,

$$
\varrho(x+y) \leq \varrho(x)+\varrho(y),
$$


for any $x, y \in \ell_{p(\cdot)}$, then we can show that the periodic point discovered in Theorem 3 is in fact a fixed point. It is not hard to see that $\varrho$ satisfies the triangle inequality if and only if $p(n)=1$, for any $n \in \mathbb{N}$.

Author Contributions: A.A.N.A. and M.A.K. contributed equally on the development of the theory and their respective analysis. Both authors have read and agreed to the published version of the manuscript.

Funding: University of Jeddah research project No. UJ-20-DR-143.

Acknowledgments: This project was funded by the University of Jeddah, Saudi Arabia, under grant No. UJ-20-DR-143. The authors, therefore, gratefully acknowledge with thanks the University technical and financial support.

Conflicts of Interest: The authors declare no conflict of interest.

\author{
Abbreviations \\ The following abbreviations are used in this manuscript: \\ MDPI Multidisciplinary Digital Publishing Institute \\ DOAJ Directory of open access journals \\ TLA Three letter acronym \\ LD linear dichroism
}

\title{
References
}

1. Aoyama, K.; Kimura, Y.; Takahashi, W. Maximal monotone operators and maximal monotone functions for equilibrium problems. J. Convex Anal. 2008, 15, 395-409.

2. Ariza-Ruiz, D.; López-Acedo, G.; Martin-Marquez, V. Firmly nonexpansive mappings. J. Nonlinear Convex Anal. 2014,15 , 1-27.

3. Bauschke, H.H.; Combettes, P.L. Convex Analysis and Monotone Operator Theory in Hilbert Spaces; Springer: New York, NY, USA, 2011.

4. Bruck, R.E.; Reich, S. Nonexpansive projections and resolvents of accretive operators in Banach spaces. Houst. J. Math. 1977, 3, 459-470.

5. Reich, S. Extension problems for accretive sets in Banach spaces. J. Funct. Anal. 1977, 26, 378-395. [CrossRef]

6. Minty, G.J. Monotone (nonlinear) Oper. Hilbert Space. Duke Math J. 1962, 29, 341-346. [CrossRef]

7. Minty, G.J. Monotonicity Gradient A Convex Function. Pac. J. Math. 1964, 14, 243-247. [CrossRef]

8. Bruck, R.E. Nonexpansive projections on subsets of Banach spaces. Pac. J. Math. 1973, 47, 341-355. [CrossRef]

9. Kaczor, W. Fixed Points of $\lambda$-firmly Nonexpansive Mappings on Nonconvex Sets. Nonlinear Anal. 2001, 47, 2787-2792. [CrossRef]

10. Smarzewski, R. On Firmly Nonexpansive Mappings. Proceeding AMS 1991, 113, 723-725. [CrossRef]

11. Khan, S.H. Approximating fixed points of $(\lambda, \rho)$-firmly nonexpansive mappings in modular function spaces. Arab. J. Math. 2018, 7, 281-287. [CrossRef]

12. Orlicz, W. Über Konjugierte Exponentenfolgen. Stud. Math. 1931, 3, 200-211. [CrossRef]

13. Klee, V. Summability in $\ell\left(p_{11}, p_{21}, \cdots\right)$ Spaces. Stud. Math. 1965, 25, 277-280. [CrossRef]

14. Nakano, H. Topology of Linear Topological Spaces; Maruzen Co. Ltd.: Tokyo, Japan, 1951.

15. Sundaresan, K. Uniform convexity of Banach spaces $\ell\left(\left\{p_{i}\right\}\right)$. Stud. Math. 1971, 39, 227-231. [CrossRef]

16. Waterman, D.; Ito, T.; Barber, F.; Ratti, J. Reflexivity and Summability: The Nakano $\ell\left(p_{i}\right)$ Spaces. Stud. Math. 1969, 331, 141-146. [CrossRef]

17. Nakano, H. Modulared Semi-Ordered Linear Spaces; Maruzen Co.: Tokyo, Japan, 1950.

18. Diening, L.; Harjulehto, P.; Hästö, P.; Ružička, M. Lebesgue and Sobolev Spaces with Variable Exponents; Lecture Note in Mathematics 2017; Springer: Berlin/Heidelberg, Germany, 2011.

19. Rajagopal, K.; Ružička, M. On the modeling of electrorheological materials. Mech. Res. Comm. 1996, 23, 401-407. [CrossRef]

20. Ružička, M. Electrorheological Fluids: Modeling and Mathematical Theory; Lecture Notes in Mathematics; Springer: Berlin/Heidelberg, Germany, 2000; Volume 1748.

21. Bansevicius, R.; Virbalis, J.A. Two-dimensional Braille readers based on electrorheological fluid valves controlled by electric field. Mechatronics 2007, 17, 570-577. [CrossRef]

22. Chen, J.Z.; Liao, W.H. Design, testing and control of a magnetorheological actuator for assistive knee braces. Smart Mater. Struct. 2010, 19, 035029. [CrossRef]

23. Choi, S.H.; Kim, S.; Kim, P.; Park, J.; Choi, S.B. A new visual feedback-based magnetorheological haptic master for 174 robotassisted minimally invasive surgery. Smart Mater. Struct. 2015, 24, 065015. [CrossRef]

24. Spencer, B.; Yang, G.; Carlson, J.; Sain, M. Smart Dampers for Seismic Protection of Structures: A Full-Scale Study. In Proceedings of the Second World Conference on Structural Control, Kyoto, Japan, 28 June-1 July 1998. 
25. Abdou, A.A.; Khamsi, M.A. On modular firmly nonexpansive mappings in the variable exponent sequence spaces $\ell_{p(.)}$. J. Fixed Point Theory Appl. 2021, 23, 8. [CrossRef]

26. Goebel, K.; Reich, S. Uniform Convexity, Hyperbolic Geometry, and Nonexpansive Mappings; Marcel Dekker: New York, NY, USA; Basel, Switzerland, 1984.

27. Khamsi, M.A.; Kirk, W.A. An Introduction to Metric Spaces and Fixed Point Theory; John Wiley: New York, NY, USA, 2001.

28. Khamsi, M.A.; Kozlowski, W.M. Fixed Point Theory in Modular Function Spaces; Birkhauser: New York, NY, USA, 2015.

29. Nakano, H. Modulared sequence spaces. Proc. Jpn. Acad. 1951, 27, 508-512. [CrossRef]

30. Khamsi, M.A.; Kozlowski, W.M.; Reich, S. Fixed point theory in modular functions spaces. Nonlinear Anal. 1990, 14, 935-953. [CrossRef]

31. Bachar, M.; Bounkhel, M.; Khamsi, M.A. Uniform Convexity in $\ell_{p(.)}$. J. Nonlinear Sci. Appl. 2017, 10, 5292-5299. [CrossRef]

32. Bachar, M.; Khamsi, M.A.; Mendez, O.; Bounkhel, M. A geometric property in $\ell_{p(\cdot)}$ and its applications. Math. Nachrichten 2019, 292, 1931-1940. [CrossRef] 